\title{
Standard-Model Higgs Boson Searches at CDF in Run II
}

\author{
Shan-Huei S. Chuang ${ }^{\mathrm{a}}$ on Behalf of the CDF Collaboration \\ ${ }^{a}$ Department of Physics, University of Wisconsin - Madison, \\ Chamberlin Hall, 1150 University Avenue, Madison WI 53706, U.S.A.
}

The SM Higgs boson has been searched in two channels: (1) $q \bar{q} \rightarrow W H \rightarrow \ell \nu b \bar{b}$ for Higgs masses $110<M_{H}<$ $150 \mathrm{GeV}$ and (2) $g g \rightarrow H \rightarrow W W \rightarrow \ell \nu \ell \nu$ for $140<M_{H}<180 \mathrm{GeV}$, where $\ell \in\{e, \mu\}$, using about $200 \mathrm{pb}^{-1}$ CDF Run II data. 95\% C.L. limits on each Higgs production at TeVatron at $\sqrt{s}=1.96 \mathrm{TeV}$ were set as a function of Higgs mass by fitting the distribution of dijet mass (1) and dilepton azimuthal angular separation (2). We have significantly advanced the sensitivity to the SM Higgs boson $c f$. Run I.

\section{Introduction}

In the Standard Model (SM) and its supersymmetric (SUSY) extensions, the Higgs boson is crucial to our understanding of electroweak symmetry breaking dynamics and the mass generation of electroweak gauge bosons and fermions. The Higgs boson mass is in addition an indicator of the scale of new physics. Electroweak precision measurements and LEP direct searches have constrained the Higgs mass to be within 114.4 and $251.1 \mathrm{GeV}$ [1]. However, while almost all the high energy particle physics phenomena observed so far have been successfully explained by the Standard Model, the Higgs boson remains unobserved. It is the last missing piece and the most important one.

\section{Higgs at TeVatron}

TeVatron is currently the only machine capable of probing the Higgs sectors in the world, of which the luminosity delivery has surpassed the design goal for FY2004. Higgs reach of TeVatron had been evaluated in [2] and [3], Figure 1, and concluded hopeful with the Run II end goal (8 $\mathrm{fb}^{-1}$ ) integrated luminosity and full strengths of both upgraded CDF and D $\varnothing$ experiments. CDF II has great improvement on energy measurement and dijet mass resolution over Run I [4]. The capabilities of CDF II on lepton ID and $b$-tagging can be found in [5] and [6]. Using data cumu-

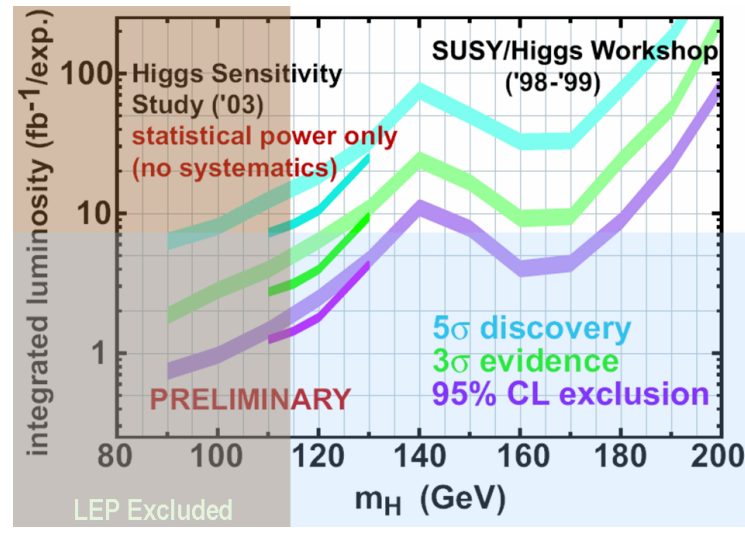

Figure 1. The integrated luminosity required per experiment to achieve $95 \%$ C.L. exclusion, $3 \sigma$ evidence or $5 \sigma$ discovery as a function of the SM Higgs mass. The thicker curves are from [2] while the thinner curves are from [3]. The brown shade indicates the LEP-excluded range while the lightblue shade indicates the TeVatron Run II possible reach.

lated so far CDF and $D \varnothing$ can already probe the SM Higgs sector with precision measurement of electroweak parameters, such as top quark mass and $W$ boson mass, Figure 2 .

\section{3. $W H \rightarrow \ell \nu b \bar{b}$ Search at CDF II}

CDF has searched for new particles decaying into $b \bar{b}$ and produced in association with $W$ 


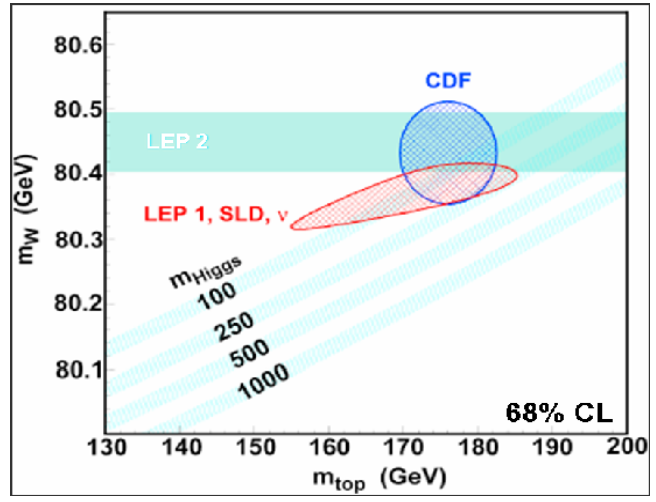

Figure 2. Higgs mass constraint obtained from top mass and $W$ mass measurements, related by $\delta M_{W} \propto\left(M_{\mathrm{top}}^{2}, \ln \left(M_{H}\right)\right)$.

bosons decaying into $\ell \nu$, where $\ell \in\{e, \mu\}$, in $p \bar{p}$ collisions at $\sqrt{s}=1.96 \mathrm{TeV}$ [7]. Such new particles could be the SM Higgs bosons if with zero spin. The final state in search consists of one high- $p_{t}$ lepton, large missing transverse energy $\not_{t}$ and two $b$ jets.

\subsection{Data Samples and Event Selection}

This analysis was based on $162 \mathrm{pb}^{-1}$ of TeVatron Run II data recorded by the CDF II detector [4] from March 2002 to September 2003, with an inclusive lepton trigger that requires an electron with $E_{t}>18 \mathrm{GeV}$ or a muon with $p_{t}>18 \mathrm{GeV}$. From the inclusive lepton dataset, events reconstructed with

1. an isolated non-conversion electron with $E_{t}>20 \mathrm{GeV}$ in the central region or noncosmic muon with $p_{t}>20 \mathrm{GeV}$;

2. $\not t_{t}>20 \mathrm{GeV}$;

3. two jets with $E_{t}>15 \mathrm{GeV}$ (cuts optimized for the best $\mathrm{S} / \sqrt{\mathrm{B}}$ ) and $|\eta|<2$, with at least one jet $b$-tagged with CDF secondary vertex tagging algorithm (SECVTX) [6] to improve signal against $W+$ jets background;

were selected. To reduce top-contributed backgrounds, events with an isolated track having $p_{t}{ }^{\text {seed }}>20 \mathrm{GeV}$ and charge opposite to the primary lepton's were removed. Events with additional jets (one with $E_{t}>8 \mathrm{GeV}$ and $2<|\eta|<3$ or two with $8<E_{t}<15 \mathrm{GeV}$ and $|\eta|<2$ ) were also removed.

\subsection{Background}

The dominant background was QCD production of $W+$ jets, which entered the signal sample when either one of the jets was $b$-tagged or a light-quark jet was mis-identified as a heavy one.

Mistags were mostly due to symmetric resolution effects and were estimated by

1. parameterizing the negative tag rate in the generic QCD multijet sample as a function of jet $E_{t}$, track multiplicity, $\eta, \phi$ and the event sum of jet $E_{t} \mathrm{~s}$. The negative tag rate was enlarged by $1.2 \pm 0.1$ to account for the presence of long-lived particles and secondary interactions with detector material;

2. applying the predicted tag rates to the pretag sample.

Fraction of $W+$ heavy-flavour jets in the inclusive $W+$ jets sample was estimated using Alpgen [8] Monte Carlo. Background of $W+$ heavyflavour jets was estimated by applying the estimated fraction and the tagging efficiency to the pretag sample.

Another substantial background came from mis-measured QCD events, in which one jet was mis-identified as a high- $p_{t}$ lepton and mismeasured energies made apparently large $\not_{t}$. To estimate this background, the number of tagged events with an isolated lepton and low $\not_{t}$ was extrapolated into the signal region.

Top-contributed backgrounds were estimated using PYTHIA [9] and assuming NLO crosssections. Other backgrounds were estimated using suitable MC.

Summary of tagged data events and expected backgrounds as a function of jet multiplicity is in Table 1, showing good agreement between data and expectation.

\subsection{Results}

\subsubsection{Acceptance and Uncertainties}

Signal acceptance was estimated using both data and PYTHIA MC, Figure 3. The total acceptance was calculated as a product of geometric and kinematic acceptance, lepton ID, trigger 


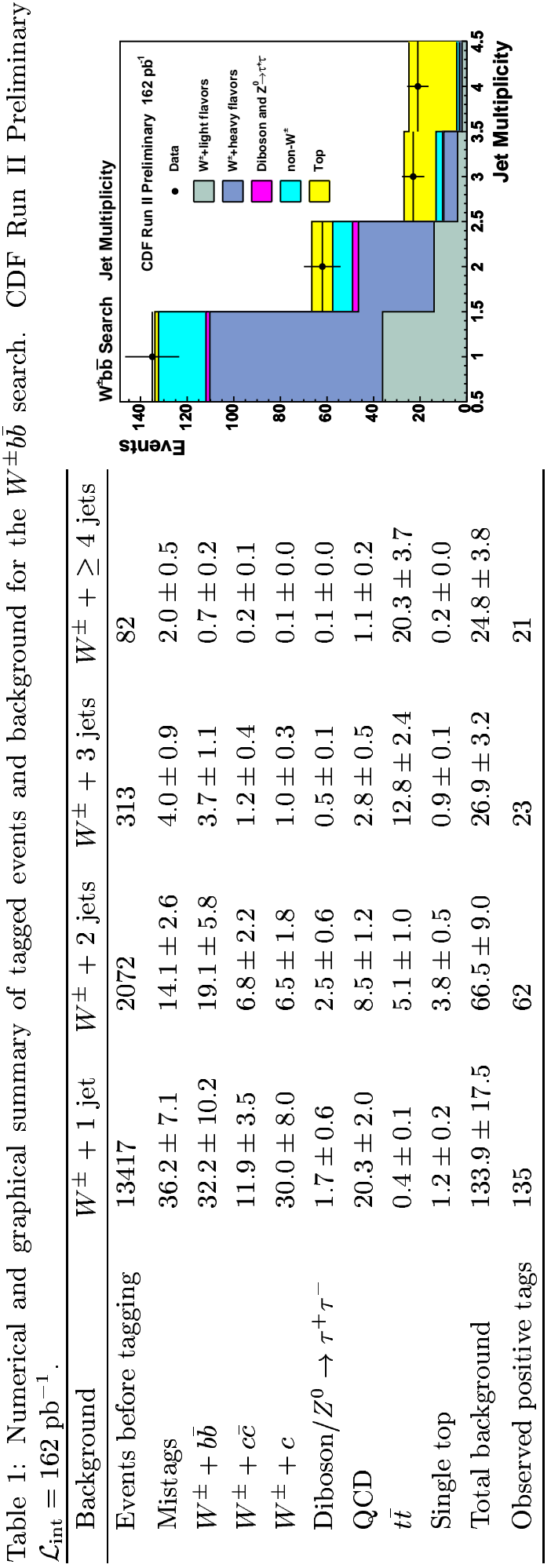

and $b$-tagging efficiencies. Systematic uncertainty (11\% in total) came from the modelling of initial $(3 \%)$ and final $(6 \%)$ state radiation, parton distribution function $(2 \%)$, jet energy scale $(3 \%)$ and resolution (1\%), $b$-tagging $(6 \%)$, lepton trigger $(<1 \%)$ and ID $(5 \%)$ efficiencies.

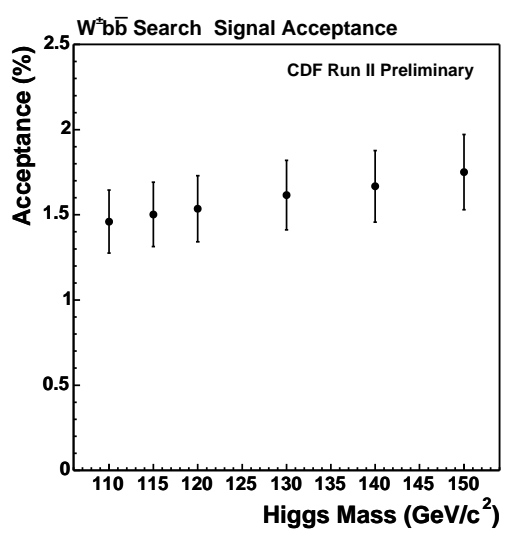

Figure 3. The total $W H \rightarrow \ell \nu b \bar{b}$ acceptance as a function of the Higgs mass.

\subsubsection{5\% C.L. Limits}

A direct search for a resonant mass peak in the reconstructed dijet mass distribution did not yield any positive result, Figure 4 . So 95\% C.L. limits on $\sigma \cdot \operatorname{BR}(p \bar{p} \rightarrow W H \rightarrow \ell \nu b \bar{b})$ were set as a function of the Higgs mass using a binned maximum likelihood technique, Equation (1), on the numbers from the $W+2$ jets bin.

$\mathcal{L} \equiv \Pi_{i} \frac{\mu_{i}^{N_{i}} \cdot e^{-\mu_{i}}}{N_{i} !}$

where $\mu_{i}$ was the expected signal plus background and $N_{i}$ was the number of observed events from the $W+2$ jets sample in the $i$ th mass bin. The limits, shown in Figure 7, were greatly improved over Run I [10] although the SM reach was still limited by statistics.

\section{4. $H \rightarrow W W \rightarrow \ell \nu \ell \nu$ Search at CDF II}

CDF has searched for the high-mass SM Higgs production and decay $p \bar{p} \rightarrow H \rightarrow W^{+} W^{-} \rightarrow$ 


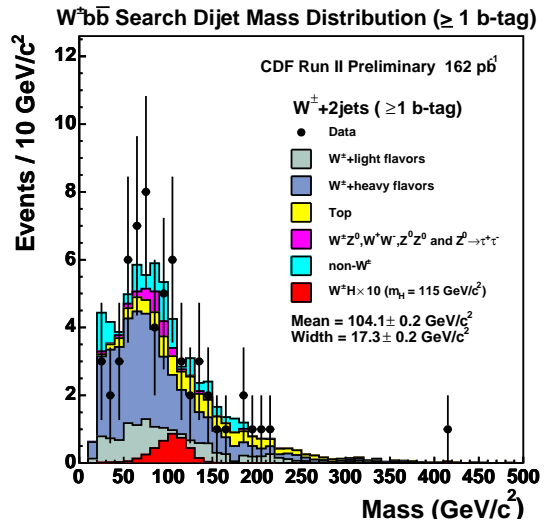

Figure 4. The dijet mass distribution of data, expected backgrounds and up-scaled $W H$ signal.

$\ell^{+} \nu \ell^{-} \bar{\nu}$ where $\ell=\{e, \mu\}$ at $\sqrt{s}=1.96 \mathrm{TeV}[11]$.

\subsection{Data Samples and Event Selection}

The analysis was based on $184 \mathrm{pb}^{-1}$ of CDF

II data collected from March 2002 to September 2003 , with one of the following inclusive high- $p_{t}$ lepton triggers:

1. a central electromagnetic cluster with $E_{t}>$ $18 \mathrm{GeV}$ and $p_{t}>9 \mathrm{GeV}$;

2. a central muon track with $p_{t}>18 \mathrm{GeV}$;

3. a forward electromagnetic cluster with $E_{t}>$ $20 \mathrm{GeV}$ and $\not_{t}>15 \mathrm{GeV}$.

The selection of a candidate event requires

1. two electron/muons with opposite charges;

2. $\not P_{t}>25 \mathrm{GeV}$;

3. no jets with $E_{t}>15 \mathrm{GeV}$ and $|\eta|<2.5$;

4. large azimuthal angular separation between small $\not_{t}$ and any lepton or jet, $\Delta \phi\left(\not_{t}, \ell / j\right)>20^{\circ}$ if $\not_{t}<50 \mathrm{GeV}$, to lower $Z \rightarrow \tau \tau$ background;

5. large $\not_{t}$ significance [12] if $\left|M_{\ell \ell}-M_{Z}\right|<$ $15 \mathrm{GeV}$, to lower $Z \rightarrow e e$ and $Z \rightarrow \mu \mu$ background;

6. small $M_{\ell \ell}{ }^{1}$, cut as shown in Table 3 .

${ }^{1}$ The $H \rightarrow W W$ signals were discriminated from the $W W$

\subsection{Acceptance}

Signal acceptance, as shown in Table 3, was estimated using PYTHIA MC with CTEQ5L parton distribution functions.

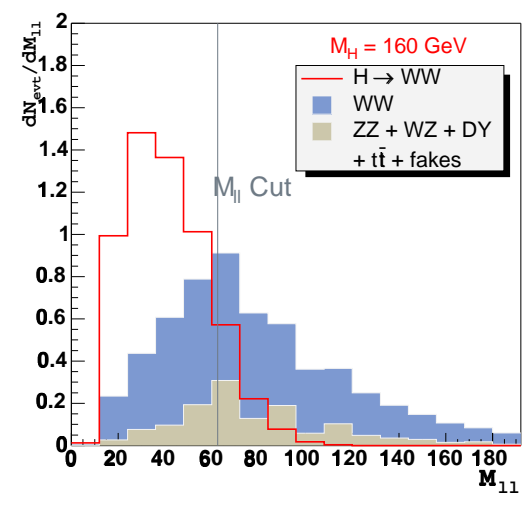

Figure 5. Dilepton invariant mass distributions of signal, $W W$ and the sum of other SM backgrounds as predicted by MC. The backgrounds were normalized to the expectation for $184 \mathrm{pb}^{-1}$ and the signal normalized to the total background expectation. The dileptons from Higgs tend to have small $M_{\ell \ell}$ while those from other SM processes extend to large $M_{\ell \ell}$.

\subsection{Background}

Drell-Yan $Z / \gamma^{*} \rightarrow \ell^{+} \ell^{-}$where $\ell=\{e, \mu, \tau\}$ and diboson $(W W, W Z \& Z Z)$ backgrounds were estimated with PYTHIA MC, $t \bar{t}$ with HERWIG [13], normalized to their SM cross-sections.

$W+$ jet entered the background when the jet was mis-identified as a lepton. The $W+$ jet background was estimated by

1. calculating the probabilities (typically of order $10^{-3}-10^{-4}$ ) for a jet to fake a lepton using the inclusive jet data;

background using the spin-zero property of the Higgs boson, which would result in small invariant mass of the two leptons $\left(M_{\ell \ell}\right)$, Figure 5, and azimuthal angular separation in between $\left(\Delta \phi_{\ell \ell}\right)$. 
Table 2

Signal and background expectations for the analyzed data. CDF Run II Preliminary $\mathcal{L}_{\text {int }}=184 \mathrm{pb}^{-1}$

\begin{tabular}{llllll}
\hline$M_{H}$ & $140 \mathrm{GeV}$ & $150 \mathrm{GeV}$ & $160 \mathrm{GeV}$ & $170 \mathrm{GeV}$ & $180 \mathrm{GeV}$ \\
\hline $\mathrm{DY} e e+\mu \mu+\tau \tau$ & $0.18 \pm 0.11$ & $0.33 \pm 0.19$ & $0.65 \pm 0.30$ & $1.06 \pm 0.42$ & $1.33 \pm 0.48$ \\
fakes & $0.40 \pm 0.12$ & $0.45 \pm 0.14$ & $0.53 \pm 0.16$ & $0.65 \pm 0.19$ & $0.81 \pm 0.25$ \\
$t \bar{t}$ & $0.01 \pm 0.01$ & $0.01 \pm 0.01$ & $0.01 \pm 0.01$ & $0.01 \pm 0.01$ & $0.02 \pm 0.01$ \\
$W Z+Z Z$ & $0.10 \pm 0.01$ & $0.13 \pm 0.01$ & $0.15 \pm 0.01$ & $0.19 \pm 0.01$ & $0.24 \pm 0.02$ \\
$W W$ & $3.50 \pm 0.41$ & $3.82 \pm 0.45$ & $4.45 \pm 0.52$ & $5.38 \pm 0.63$ & $6.49 \pm 0.76$ \\
total background & $4.19 \pm 0.45$ & $4.74 \pm 0.52$ & $5.79 \pm 0.64$ & $7.29 \pm 0.81$ & $8.89 \pm 0.98$ \\
\hline
\end{tabular}

2. counting the number of events containing a faking jet and a lepton and passing the $H \rightarrow W W$ dilepton event selection from the parent signal sample;

3. applying the fake probabilities to the number of events.

Summary of signal and background expectations for the $184^{-1}$ analyzed data is given in Table 2 and shown in Figure 6.

\subsection{Results}

The binned maximum likelihood method was used on the $\Delta \phi_{\ell \ell}$ distribution of data and MC signal, $W W$ and the sum of other SM backgrounds to set $95 \%$ C.L. upper limits on $\sigma \cdot \mathrm{BR}_{\mathrm{SM}}(p \bar{p} \rightarrow$ $H \rightarrow W W)$ as a function of the Higgs mass for $140<M_{H}<180 \mathrm{GeV}$. We compare the results to those obtained by using a simple counting method. Summary of results is in Table 3, showing good agreement between data and expectation, and Figure 7.

\section{Conclusion}

Higgs hunting Run II at CDF has started! With currently available data, we should/can set tight Higgs constraints with precision top and electroweak measurement besides getting exciting first Higgs direct search results. Eventually we will likely make sensitive tests of the SM Higgs sector with the Run II end goal luminosity of TeVatron and full strengths of both CDF and $\mathrm{D} \emptyset$ experiments.

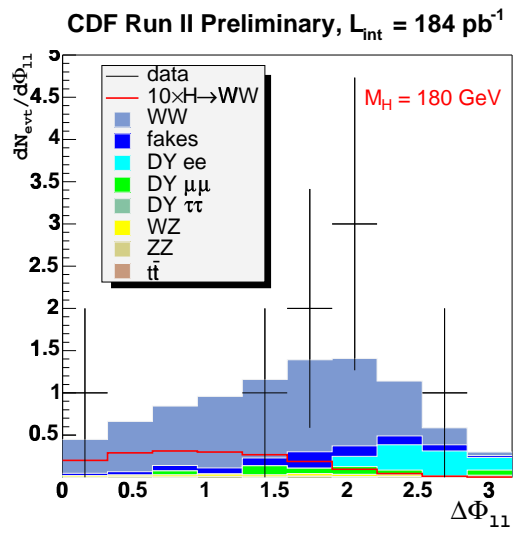

Figure 6. The dilepton azimuthal angular separation $\Delta \phi_{\ell \ell}$ distribution of data and MC signal and background expectations.

\section{REFERENCES}

1. hep-ex/0306033, Phys. Lett. B 565: 61-75.

2. hep-ph/0010338.

3. FERMILAB-PUB-03-320-E.

4. Phys. Rev. D 52, 4784; Nucl. Instrum. Meth. Phys. Res. A 360, 137; The CDF II Detector Technical Design Report, Fermilab-Pub96/390-E.

5. hep-ex/0406078, submitted to PRL.

6. Measurement of the $t \bar{t}$ Cross Section using Lepton + Jets Events with Secondary Vertex $b$-Tagging, PRD in preparation.

7. CDF/PUB/EXOTIC/PUBLIC/7126.

8. hep-ph/0206293, JHEP 0307: 001.

9. Comput. Phys. Commun. 135, 238. 


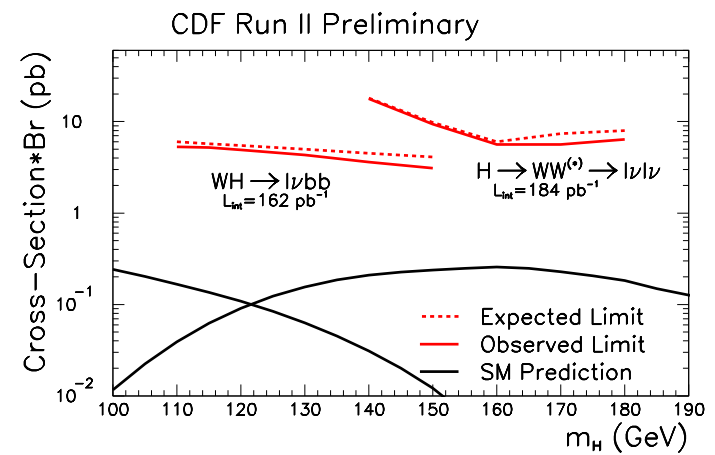

Figure 7. CDF Run II 95\% C.L. and expected upper limits for $W H$ and $H \rightarrow W W$ production at TeVatron, in comparison with the StandardModel predictions.

10. Phys. Rev. Lett. 79, 3819;

Phys. Rev. Lett. 81, 5748;

http://home.fnal.gov/\%7Emkruse/vh_combined.html

11. CDF/DOC/EXOTIC/PUBLIC/7152.

12. CDF/DOC/ELECTROWEAK/PUBLIC/7135.

13. Comput. Phys. Commun. 67: 465-508; JHEP 0101: 010. 